\title{
Stereocontrolled syntheses of 2,5-disubstituted tetrahydrofurans using remote asymmetric induction
}

\author{
Lindsay A. Hobson, L. Warjeet Singh, and Eric J. Thomas* \\ The Department of Chemistry, The University of Manchester, Manchester, M13 9PL, UK \\ E-mail:e.j.thomas@man.ac.uk
}

Dedicated to Professor T. R. Govindachari on the occasion of his $85^{\text {th }}$ birthday
(received 12 Apr 01; accepted 21 Aug 01; published on the web 29 Aug 01)

\begin{abstract}
Stereocontrolled syntheses of cis- and trans-2,5-disubstituted tetrahydrofurans with a 1-methyl2-benzyloxyethyl group at $\mathrm{C}(2)$ are described. The starting material for this work was prepared by the tin(IV) chloride promoted reaction of a 4-methyl-5-benzyloxypent-2-enylstannane with an aldehyde which proceeds with excellent 1,5-induction.
\end{abstract}

Keywords : Tetrahydrofuran, stereoselective, cyclization, homoallylic alcohol, allylstannanes

\section{Introduction}

Several biologically active natural products are characterized by the presence of cis-2,5disubstituted tetrahydrofurans which have an $\alpha$-methyl bearing stereogenic centre in the 2substituent. Examples include the pamamycins, e.g. pamamycin $607 \mathbf{1}^{1}$ and nonactic acid 2. During recent studies on the reactions of allylstannanes with aldehydes, it was found that the 4methyl-5-benzyloxypent-2-enylstannane 3 was transmetallated stereoselectively to give an allyltin trichloride which reacted with aldehydes with excellent 1,5-stereocontrol to give the (Z)1,5-anti-alkenols 4. If procedures could be developed for the stereoselective cyclisation of these alkenols to 2,5-cis-disubstituted tetrahydrofurans 5, then it may be possible to develop this strategy to provide total syntheses of nonactic acid and pamamycin 607 . 

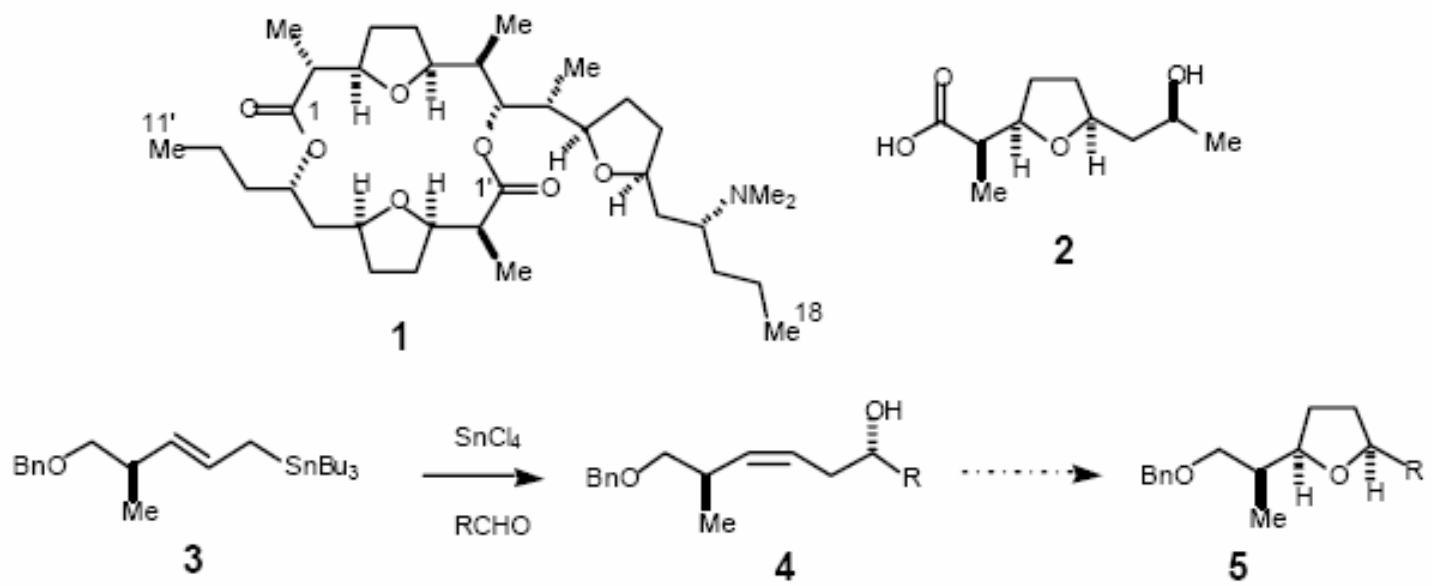

Early studies into the preparation of the tetrahydrofurans 5 from the alkenols $\mathbf{4}$, were based on dehydration/cyclisation of hydroxyselenides prepared by epoxidation of the alkenols followed by epoxide ring opening using sodium phenylselenide. ${ }^{4,5}$ However, this chemistry was successful only with relatively simple systems. With more complex substrates, the dehydration/cyclisation step was accompanied by loss of phenylselenenic acid giving the returned alkenol as the major product. $^{6}$ It transpired that direct cyclisation using either phenylselenenyl chloride ${ }^{7}$ or phthalimide $^{8}$ in the presence of $20 \mathrm{~mol} \%$ of tin(IV) chloride was more successful and led to the completion of syntheses of the methyl ester of nonactic acid 2 and pamamycin 6071 . ${ }^{9}$ During the course of this work it was necessary to prepare a sample of a cis-2,5-disubstituted tetrahydrofuran by a stereochemically unambiguous route. The results of this study are described herein together with procedures for the synthesis of the diastereoisomeric 2,5-trans-isomers.

\section{Results and Discussion}

The reaction between butanal and the allyltin trichloride generated from the 4-methyl-5benzyloxypent-2-enylstannane 3 gave the (Z)-1,5-anti-alkenol 6 with excellent stereocontrol, 1,5-anti : 1,5-syn $\geq 96: 4$, with no (E)-alkenols being detected (Scheme 1). ${ }^{3}$ It was decided that one strategy for tetrahydrofuran formation would be to effect regio- and stereoselective hydration of the double-bond and so the alkenol 6 was converted into the syn-epoxide 7 using the wellprecedented vanadyl catalysed synepoxidation procedure. ${ }^{10}$ This epoxidation was highly stereoselective with only the one stereoisomer being detected $(\geq 95: 5)$ in the crude reaction mixture by high field ${ }^{1} \mathrm{H}$ NMR. Since this stereoselectivity is higher than that usually observed in this kind of epoxidation (more typically $85: 15$ in favour of the syn-diastereoisomer), it may well be that both of the stereogenic centres in the alkenol contribute to this stereocontrol. 


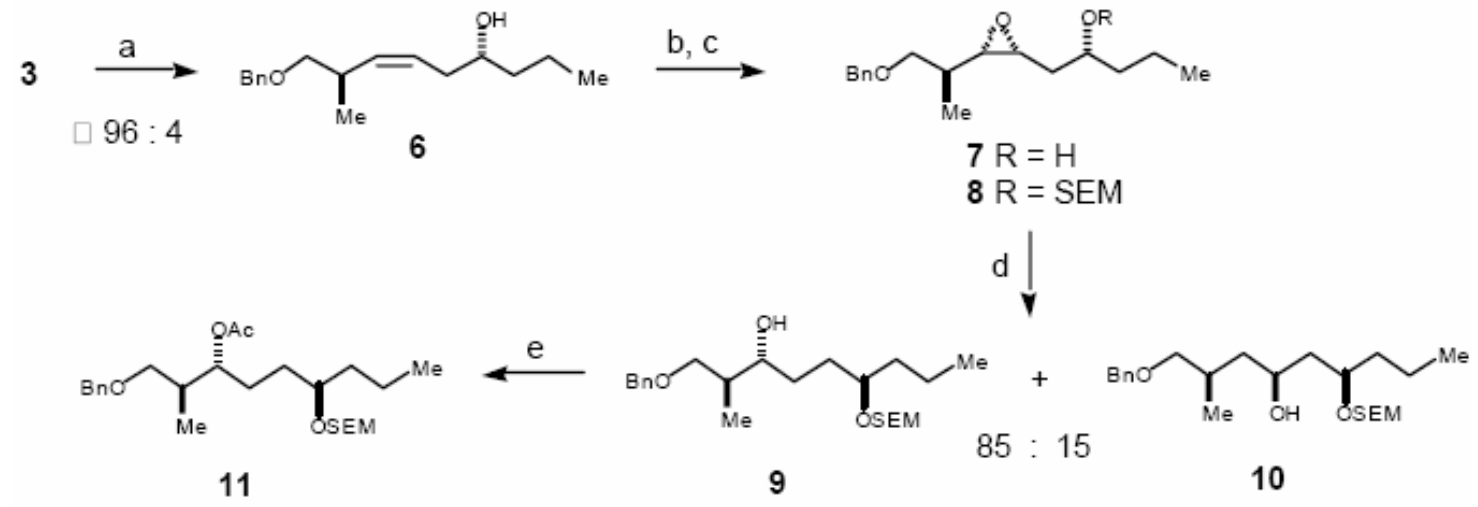

Scheme 1. (a) $\mathrm{SnCl}_{4},-78{ }^{\circ} \mathrm{C}, 5 \mathrm{~min}, n-\mathrm{PrCHO}$ (78\%); (b) $\mathrm{VO}(\mathrm{acac})_{2}, t-\mathrm{BuOOH}(80 \%$ ); (c) SEMCl, i-Pr ${ }_{2} \mathrm{NEt}$ (80\%); (d) Red-Al, 15 h (52\%); (e) $\mathrm{Ac}_{2} \mathrm{O}, \mathrm{Et}_{3} \mathrm{~N}, \mathrm{DMAP}$ (48\%).

The next step was to reduce the epoxide regioselectively to give a 1,4-difunctionalised intermediate as the precursor of the tetrahydrofuran ring. To avoid problems of having to differentiate between two secondary hydroxyl groups later in the synthesis, the hydroxy-epoxide 7 was first converted into its SEM-ether 8. Reduction of this epoxyether by Red-Al was found to be usefully regioselective and gave the required alcohol $\mathbf{9}$ together with its regioisomer $\mathbf{1 0}$ in a ratio of $c a .85: 15$. The regioselectivity of this reduction was established by a COSY ${ }^{1} \mathrm{H} N M R$ study of the acetate $\mathbf{1 1}$ of the major reduction product $\mathbf{9}$ and may be due to reduced steric hindrance to attack at $\mathrm{C}(4)$ and preferred co-odination by the OSEM substituent.

It now remained to convert the hydroxyl group of the alcohol $\mathbf{9}$ into a good leaving group with inversion of configuration. Preliminary studies into the preparation of its inverted mesylate 12 using a Mitsunobu reaction were not promising since only low conversion into product was observed at room temperature and extensive decomposition took place in toluene heated under reflux (Scheme 2). However, reaction with iodine, imidazole and triphenylphosphine gave the inverted iodide 13 in a reasonable, non-optimized yield of 64\%. Removal of the SEM-protecting group using dilute aqueous hydrogen fluoride gave mainly the iodo-alcohol $\mathbf{1 4}$ together with a small amount of the tetrahydrofuran 15. Conversion of the remainder of the iodo-alcohol into the tetrahydrofuran was accomplished by treatment with sodium hydride in THF as solvent and gave the 2,5-cis-disubstituted tetrahydrofuran 15 in a 65\%yield.

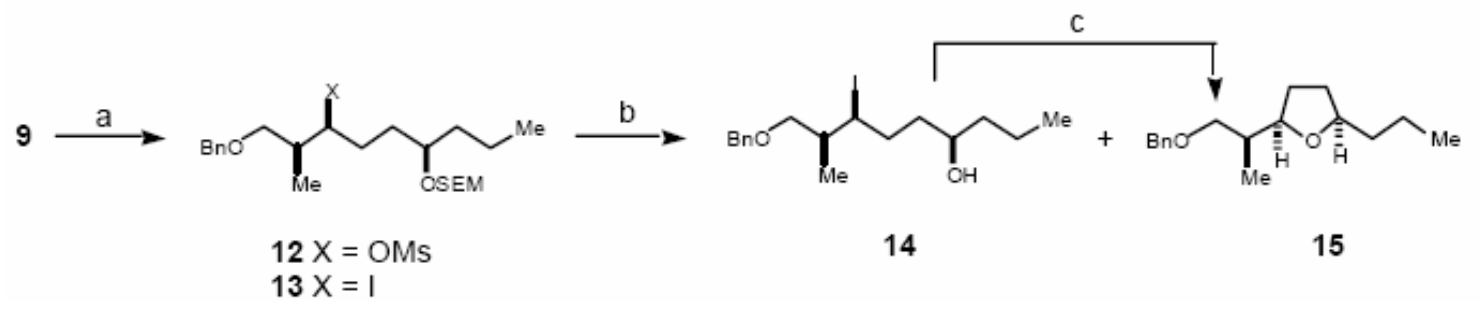

Scheme 2. (a) $\mathrm{I}_{2}, \mathrm{Ph}_{3} \mathrm{P}$, imid. (64\%) (b) 40\% HF, acetonitrile (14, 57\%; 15, 18\%); (c) $\mathrm{NaH}$, THF $(65 \%)$. 
The 1,5-cis-configuration assigned to the tetrahydrofuran 15 follows from its method of synthesis and was confirmed by the synthesis of the racemic 1,5-trans-isomer 17 (Scheme 3). Thus deprotection of the mesylate $\mathbf{1 6}$ prepared from the racemic alcohol $\mathbf{9}$ was accompanied by cyclisation and gave the 2,5-trans-disubstituted tetrahydrofuran 17 directly. The 2,5-cis- and 2,5-trans-disubstituted tetrahydrofurans 15 and 17 could be distinguished spectroscopically. These syntheses of the tetrahydrofurans $\mathbf{1 5}$ and $\mathbf{1 7}$ were found to be highly stereoselective with little, if any $(\leq 5 \%)$, contamination by the other stereoisomer being observed.

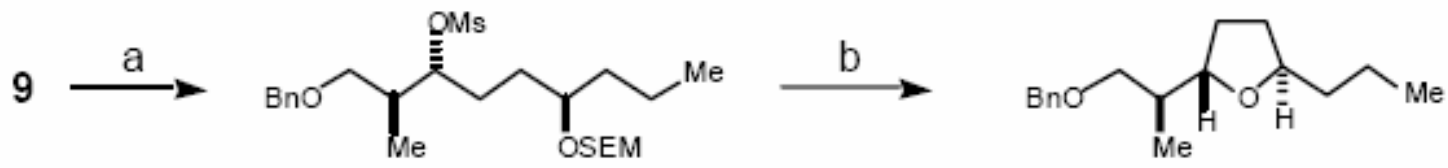

16

17

Scheme 3. (a) $\mathrm{MsCl}, \mathrm{Et}_{3} \mathrm{~N}$, (61\%); (b) 40\% HF, acetonitrile (31\%).

A second synthesis of the racemic 2,5-trans-isomer 17 from the hydroxyepoxide 7 was also developed. Thus treatment of the hydroxyepoxide with tert-butyldimethylsilyl triflate and trimethylaluminium gave a mixture of the hydroxytetrahydrofurans $\mathbf{1 8}$ and $\mathbf{1 9 .}{ }^{11}$ The stereochemical homology between these two compounds was proved by their deprotection which gave the same diol 20. Removal of the hydroxyl group from 19 was accomplished by conversion into the thionocarbonate 21 which, on reduction using tributyltin hydride, gave the 2,5-transdisubstituted terahydrofuran $\mathbf{1 7}$ identical to a sample prepared by the earlier procedure (Scheme 4).

This work shows how the alkenols prepared with 1,5-stereocontrol using the allylstannane 3 can be incorporated into stereoselective syntheses of either 2,5-cis- or trans-disubstituted tetrahydrofurans. The synthesis of natural products using this and related chemistry is underway. ${ }^{9}$

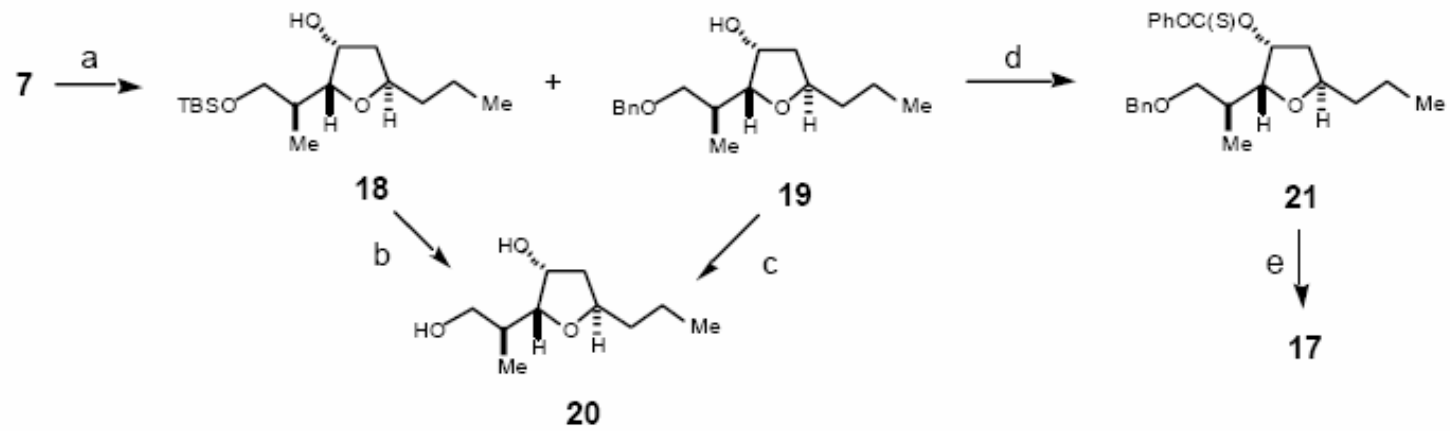

Scheme 4. (a) TBSOTf, Me3Al (18, 22\%; 19, 63\%); (b) TBAF (89\%); (c) $\mathrm{H}_{2}, \mathrm{Pd} / \mathrm{C}(91 \%)$; PhOC(S)Cl, py (83\%); (e) Bu3SnH, AIBN (53\%). 


\section{Experimental Section}

General Procedures. ${ }^{1} \mathrm{H}$ and ${ }^{13} \mathrm{C}$ NMR spectra were recorded on Bruker AC 300, Varian Inova 300 and Varian Gemini 200 spectrometers in chloroform- $d_{1}$. Mass spectra were recorded on Kratos Concept 1S and Fisons VG Trio 2000 mass spectrometers using electron impact (EI) or chemical ionisation (CI) modes. IR spectra were recorded on an ATI Mattson Genesis FTIR spectrometer as evaporated films on sodium chloride plates unless otherwise stated. Flash column chromatography was carried out using Merck silica gel $60 \mathrm{H}$ (40-60 $\mu, 230-300$ mesh) as the stationary phase. Optical rotations were measured on an Optical Activity AA-100 polarimeter operating at $589 \mathrm{~nm}$. Light petroleum refers to the fraction with b.p. $40^{\circ} \mathrm{C}-60^{\circ} \mathrm{C}$ and was redistilled before use. Ether refers to diethyl ether. All solvents were distilled and purified by standard procedures. All products were obtained as colourless oils after chromatography.

(4R,8R,6Z)-9-(Benzyloxy)-8-methyl-6-nonen-4-ol (6). A cooled solution, $-78{ }^{\circ} \mathrm{C}$, of tin(IV) chloride $\left(2.1 \mathrm{~cm}^{3}, 2.1 \mathrm{mmol}\right)$ in dichloromethane was added to a stirred solution of the stannane $3(1.0 \mathrm{~g}, 2.08 \mathrm{mmol})$ in dichloromethane $\left(20 \mathrm{~cm}^{3}\right)$ at $-78{ }^{\circ} \mathrm{C}$. After $5 \mathrm{~min}$, a cooled solution of butanal $\left(2.5 \mathrm{~cm}^{3}, 2.5 \mathrm{mmol}\right)$ in dichloromethane was added and the mixture was stirred at -78 ${ }^{\circ} \mathrm{C}$ for $45 \mathrm{~min}$. Saturated aqueous sodium bicarbonate $\left(20 \mathrm{~cm}^{3}\right)$ was added and the mixture was allowed to warm to room temperature. The mixture was partitioned between dichloromethane $\left(60 \mathrm{~cm}^{3}\right)$ and water $\left(60 \mathrm{~cm}^{3}\right)$ and the organic phase washed with water $\left(40 \mathrm{~cm}^{3}\right)$, brine $\left(40 \mathrm{~cm}^{3}\right)$ and dried $\left(\mathrm{MgSO}_{4}\right)$. After concentration under reduced pressure, flash chromatography of the residue using light petroleum : ether, $(3: 1)$ and triethylamine $(1 \%)$ as eluant gave the title compound, 6 (0.426 g, 78\%), as a colourless oil, [ $\alpha] \mathrm{D}^{22}-7.6$ (c 0.91, $\mathrm{CHCl}_{3}$ ); (Found: $\mathrm{M}^{+}+\mathrm{H}$, 263.2013. $\mathrm{C}_{17} \mathrm{H}_{27} \mathrm{O}_{2}$ requires $\left.M, 263.2010\right) ; v_{\max } / \mathrm{cm}^{-1} 3429,1454,1364,1094,1024,738$ and 698; $\delta_{\mathrm{H}} 0.85\left(6 \mathrm{H}, \mathrm{m}, 1-\mathrm{H}_{3}, 8-\mathrm{CH}_{3}\right), 1.37\left(4 \mathrm{H}, \mathrm{m}, 2-\mathrm{H}_{2}\right.$ and $\left.3-\mathrm{H}_{2}\right), 2.02-2.26\left(2 \mathrm{H}, \mathrm{m}, 5-\mathrm{H}_{2}\right)$, $2.72(1 \mathrm{H}, \mathrm{d}, J 3, \mathrm{OH}), 2.80(1 \mathrm{H}, \mathrm{m}, 8-\mathrm{H}), 3.10(1 \mathrm{H}, \mathrm{t}, J$ 8, 9-H), $3.25(1 \mathrm{H}, \mathrm{dd}, J 8$ and 5, 9-H), $3.51(1 \mathrm{H}, \mathrm{m}, 4-\mathrm{H}), 4.41$ and 4.47 (each $\left.1 \mathrm{H}, \mathrm{d}, J 12, \mathrm{PhCH}_{2} \mathrm{O}\right), 5.25$ (1 H, t, J 10, 7-H), 5.41 (1 $\mathrm{H}, \mathrm{m}, 6-\mathrm{H})$ and $7.18-7.30(5 \mathrm{H}, \mathrm{m}, \mathrm{Ph}-\mathrm{H}) ; \mathrm{CC} 14.0,17.2,18.8,32.2,35.7,39.4,70.5,72.9,74.6$, $126.3,127.4,127.6,128.2,136.1$ and $138.0 ; \mathrm{m} / \mathrm{z}$ (C.I.) $280\left(\mathrm{M}^{+}+18,19 \%\right), 263\left(\mathrm{M}^{+}+1,100\right)$ and 245 (14).

(2R)-1-\{(2R,3S)-3-[(1S)-2-(Benzyloxy)-1-methylethyl]oxiran-2-yl\}pentan-2-ol (7). Vanadyl ac-etoacetonate $(4 \mathrm{mg}, 2 \mathrm{~mol} \%, 0.016 \mathrm{mmol})$ and tert-butyl hydroperoxide $\left(0.24 \mathrm{~cm}^{3}, 5 \mathrm{M}\right.$ solution in nonane, $1.20 \mathrm{mmol})$ were added to a solution of the alkene $6(0.21 \mathrm{~g}, 0.801 \mathrm{mmol})$ in dichloromethane $\left(7 \mathrm{~cm}^{3}\right)$ at $0{ }^{\circ} \mathrm{C}$. After $10 \mathrm{~min}$, the solution was allowed to warm to room temperature and stirred for a further $20 \mathrm{~h}$. Saturated aqueous sodium thiosulfate $\left(5 \mathrm{~cm}^{3}\right)$ was added and the mixture extracted with ether $\left(3 \times 10 \mathrm{~cm}^{3}\right)$. The combined organic phase was washed with water $\left(10 \mathrm{~cm}^{3}\right)$, brine $\left(10 \mathrm{~cm}^{3}\right)$, dried $\left(\mathrm{MgSO}_{4}\right)$ and concentrated under reduced pressure. Flash chromatography of the residue using light petroleum : ether, $(2: 1)$ as eluant gave the title compound, $7(0.178 \mathrm{~g}, 80 \%)$, as a colourless oil, [ $\alpha] \mathrm{D}^{21}-10.3$ (c 0.96, $\left.\mathrm{CHCl}_{3}\right)$; (Found: $\mathrm{M}^{+}+\mathrm{NH} 4,296.2221 . \mathrm{C}_{17} \mathrm{H}_{30} \mathrm{NO}_{3}$ requires $\left.M, 296.2225\right) ; v_{\max } / \mathrm{cm}^{-1} 3436,1453,1360,1095$, 
1072, 1026, 737 and 697; $\delta_{\mathrm{H}} 0.98(3 \mathrm{H}, \mathrm{t}, J$ 7, 5-H $), 1.08\left(3 \mathrm{H}, \mathrm{d}, J\right.$ 7, 1" $\left.-\mathrm{CH}_{3}\right), 1.34$ - $1.78(6 \mathrm{H}$, m, 1-H, 3-H2, 4-H and 1"-H), 1.91 (1 H, dt, $J 7$ and 3, 1-H), 2.27 (1 H, brs, OH), 2.86 (1 H, dd, $J 9$ and 4, 3'H), 3.20 (1 H, m, 2'-H), 3.53 (1 H, dd, $J 9$ and 6, 2"-H), 3.64 (1 H, dd, $J 9$ and 4, 2"$\mathrm{H}), 3.98(1 \mathrm{H}, \mathrm{m}, 2-\mathrm{H}), 4.58\left(2 \mathrm{H}, \mathrm{s}, \mathrm{PhCH}_{2} \mathrm{O}\right)$ and $7.30-7.40(5 \mathrm{H}, \mathrm{m}, \mathrm{Ph}-\mathrm{H}) ; \delta_{\mathrm{C}} 13.5,13.9$, 18.6, 33.2, 34.6, 39.5, 54.8, 57.8, 70.6, 73.1, 127.4, 128.2 and 138.4; $\mathrm{m} / \mathrm{z}$ (C.I.) $296\left(\mathrm{M}^{+}+18\right.$, $100 \%), 279\left(\mathrm{M}^{+}+1,60\right)$ and $207(19)$.

[2-(\{[(1R)-1-(\{(2R,3S)-3-[(1S)-2-(Benzyloxy)-1-methylethyl]oxiran-2-yl\}methyl)butyl]oxy\}methoxy)ethyl](trimethyl)silane (8). DIPEA $\left(1.64 \mathrm{~cm}^{3}, 9.39 \mathrm{mmol}\right)$ was added to a solution of epoxide $7(0.87 \mathrm{~g}, 3.12 \mathrm{mmol})$ in dichloromethane $\left(5 \mathrm{~cm}^{3}\right)$ at $0{ }^{\circ} \mathrm{C}$. After $20 \mathrm{~min} \mathrm{SEM-Cl}$ $\left(0.83 \mathrm{~cm}^{3}, 4.69 \mathrm{mmol}\right)$ was added and the resultant solution was stirred at room temperature overnight. The reaction was quenched by the addition of water $\left(3 \mathrm{~cm}^{3}\right)$. The organic phase was separated and washed with water $\left(3 \mathrm{~cm}^{3}\right)$, brine $\left(3 \mathrm{~cm}^{3}\right)$, dried $\left(\mathrm{MgSO}_{4}\right)$ and the solvent was removed under reduced pressure to leave an oil which was purified by flash chromatography using light petroleum : ether, (4 : 1), as eluant. The title compound, 8 (1.03 g, 80\%), was obtained as a colourless oil, $[\alpha]_{\mathrm{D}}{ }^{22}-7.5$ (c 1.05, $\mathrm{CHCl}_{3}$ ); (Found: $\mathrm{M}^{+}+\mathrm{NH}_{4}, 426.3044$. $\mathrm{C}_{23} \mathrm{H}_{40} \mathrm{NO}_{4} \mathrm{Si}$ requires $\left.M, 426.3039\right) ; v_{\max } / \mathrm{cm}^{-1} 1455,1362,1248,1194,1099,1052,1032,859$, 836, 738 and 696; $\delta_{\mathrm{H}} 0.00\left(9 \mathrm{H}, \mathrm{s},\left(\mathrm{CH}_{3}\right)_{3} \mathrm{Si}\right), 0.92\left(5 \mathrm{H}, \mathrm{m}, 4-\mathrm{H} 3\right.$ and $\left.\mathrm{CH}_{2} \mathrm{Si}\right), 1.05(3 \mathrm{H}, \mathrm{d}, J$, $\left.1^{\prime \prime}-\mathrm{CH}_{3}\right), 1.20-1.90$ (7 H, m, 2-H $, 3-\mathrm{H}_{2}, 1^{\prime}-\mathrm{H}_{2}$, and $\left.1^{\prime \prime}-\mathrm{H}\right), 2.76$ (1 H, dd, J 9 and 4, 3'-H), 3.09 $\left(1 \mathrm{H}, \mathrm{m}, 2^{\prime}-\mathrm{H}\right), 3.48$ (1 H, dd, $J 9$ and 7, 2"-H), $3.62\left(3 \mathrm{H}, \mathrm{m}, 2^{\prime \prime}-\mathrm{H}\right.$ and $\left.\mathrm{OCH}_{2} \mathrm{CH} 2 \mathrm{Si}\right), 3.78(1 \mathrm{H}$, $\mathrm{m}, 1-\mathrm{H}), 4.54\left(2 \mathrm{H}, \mathrm{s}, \mathrm{PhCH}_{2} \mathrm{O}\right), 4.71\left(2 \mathrm{H}, \mathrm{s}, \mathrm{OCH}_{2} \mathrm{O}\right)$ and 7.24 - $7.36(5 \mathrm{H}, \mathrm{m}, \mathrm{Ph}-\mathrm{H})$; $\delta \mathrm{C}-1.5$, $13.7,14.0,18.0,18.5,32.5,33.2,36.6,53.4,57.9,65.0,73.1,73.3,75.2,93.4,127.3,127.4$, 128.2 and 138.4; $\mathrm{m} / \mathrm{z}$ (C.I.) $426\left(\mathrm{M}^{+}+18,100 \%\right), 261$ (15) and 90 (12).

(2S,3R,6R)-1-(Benzyloxy)-2-methyl-6-\{[2-(1,1,1-trimethylsilyl)ethoxy]methoxy\}-nonane-3-ol (9). Red-Al $\left(0.23 \mathrm{~cm}^{3}, 65 \% \mathrm{w} / \mathrm{v}\right.$ in toluene, $\left.0.764 \mathrm{mmol}\right)$ was added to a solution of epoxide 8 $(0.104 \mathrm{~g}, 0.254 \mathrm{mmol})$ in tetrahydrofuran $\left(2 \mathrm{~cm}^{3}\right)$ at $0{ }^{\circ} \mathrm{C}$. The solution was then heated under reflux for $15 \mathrm{~h}$. The reaction mixture was cooled to room temperature and saturated aqueous ammonium chloride $\left(2 \mathrm{~cm}^{3}\right)$ was added. The precipitate was removed by filtration and the filtrate was extracted with ether $\left(3 \times 3 \mathrm{~cm}^{3}\right)$. The organic phase was washed with water $\left(3 \mathrm{~cm}^{3}\right)$, brine $\left(3 \mathrm{~cm}^{3}\right)$, dried $\left(\mathrm{MgSO}_{4}\right)$ and the solvent was removed under reduced pressure. Flash chromatography of the residue using light petroleum : ether, $(2: 1)$ yielded the title compound, 9 (54 mg, 52\%), as a colourless oil, $[\alpha]_{\mathrm{D}}{ }^{22}$-3.69 (c 0.976, $\mathrm{CHCl}_{3}$ ); (Found: $\mathrm{M}^{+}+\mathrm{H}, 411.2936$. $\mathrm{C}_{23} \mathrm{H}_{43} \mathrm{O}_{4} \mathrm{Si}$ requires $\left.M, 411.2930\right) ; v_{\max } / \mathrm{cm}^{-1} 3460,1454,1369,1249,1098,1053,1029,926$, 859, 836, 739 and 697; $\delta_{\mathrm{H}} 0.00\left(9 \mathrm{H}, \mathrm{s},\left(\mathrm{CH}_{3}\right)_{3} \mathrm{Si}\right), 0.91\left(8 \mathrm{H}, \mathrm{m}, 2-\mathrm{CH}_{3}, 9-\mathrm{H}_{3}\right.$ and $\left.\mathrm{CH}_{2} \mathrm{Si}\right), 1.23-$ $2.06\left(9 \mathrm{H}, \mathrm{m}, 2-\mathrm{H}, 4-\mathrm{H} 2,5-\mathrm{H}_{2}, 7-\mathrm{H}_{2}\right.$ and 8- $\left.\mathrm{H}_{2}\right), 3.38-3.68\left(6 \mathrm{H}, \mathrm{m}, 1-\mathrm{H}_{2}, 3-\mathrm{H}, 6-\mathrm{H}\right.$ and $\left.\mathrm{OCH}_{2} \mathrm{CH}_{2} \mathrm{Si}\right), 4.50\left(2 \mathrm{H}, \mathrm{s}, \mathrm{PhCH}_{2} \mathrm{O}\right), 4.68\left(2 \mathrm{H}, \mathrm{s}, \mathrm{OCH}_{2} \mathrm{O}\right)$ and 7.25 - $7.37(5 \mathrm{H}, \mathrm{m}, \mathrm{Ph}-\mathrm{H})$; $\delta \mathrm{C}-$ $1.5,13.9,14.1,18.0,18.4,30.1,30.4,36.5,38.5,65.0,65.2,73.3,74.9,76.0,76.5,93.3,126.8$, 127.5, 128.3, 128.4 and 137.7; m/z (C.I.) $411\left(\mathrm{M}^{+}+1,12 \%\right), 310$ (11), 293 (100) and 90 (15).

(1R,4R)-1-[(1S)-2-(Benzyloxy)-1-methylethyl]-4-\{[2-(1,1,1-rimethylsilyl)ethoxy]methoxy\}-

heptyl) acetate (11). Acetic anhydride $(8 \mu 1,0.091 \mathrm{mmol})$ was added to a solution of alcohol $\mathbf{9}$, (30 mg, $0.073 \mathrm{mmol})$, triethylamine $(20 \mu \mathrm{l}, 0.146 \mathrm{mmol})$ and DMAP (1 $\mathrm{mg}$, cat.) in 
dichloromethane $\left(1 \mathrm{~cm}^{3}\right)$ at $0{ }^{\circ} \mathrm{C}$. The resultant solution was stirred at room temperature for $4 \mathrm{~h}$. Water $\left(1 \mathrm{~cm}^{3}\right)$ was added and the mixture was extracted with dichloromethane $\left(3 \times 2 \mathrm{~cm}^{3}\right)$. The combined organic phase was washed with saturated aqueous sodium hydrogen carbonate (3 $\left.\mathrm{cm}^{3}\right)$, brine $\left(3 \mathrm{~cm}^{3}\right)$, dried $\left(\mathrm{MgSO}_{4}\right)$ and the solvent was removed under reduced pressure. Flash chromatography using light petroleum : ether, (3:1) afforded the title compound, 11 (16 mg, $48 \%$ ) as a colourless oil, (Found: $\mathrm{M}^{+}+\mathrm{NH}_{4}, 470.3297 . \mathrm{C}_{25} \mathrm{H}_{48} \mathrm{NO}_{5}$ requires $M, 470.3301$ ); $v_{\max } /$ $\mathrm{cm}^{-1} 1736,1454,1371,1243,1099,1052,1030,835$ and $696 ; \delta_{\mathrm{H}} 0.00\left(9 \mathrm{H},\left(\mathrm{CH}_{3}\right)_{3} \mathrm{Si}\right), 0.93(8 \mathrm{H}$, $\mathrm{m}, 1^{\prime}-\mathrm{CH}_{3}, 7-\mathrm{H}_{3}$, and $\left.\mathrm{CH}_{2} \mathrm{Si}\right), 1.20-1.90\left(8 \mathrm{H}, \mathrm{m}, 2-\mathrm{H}_{2}, 3-\mathrm{H}_{2}, 5-\mathrm{H}_{2}\right.$ and $\left.6-\mathrm{H}_{2}\right), 1.99(3 \mathrm{H}, \mathrm{s}$, $\left.\mathrm{CH}_{3} \mathrm{CO}\right), 2.06\left(1 \mathrm{H}, \mathrm{m}, 1^{\prime}-\mathrm{H}\right), 3.26\left(1 \mathrm{H}, \mathrm{dd}, J 9\right.$ and $\left.7,2^{\prime}-\mathrm{H}\right), 3.44(1 \mathrm{H}, \mathrm{dd}, J 9$ and 5, 2'-H), 3.48 - $3.68\left(3 \mathrm{H}, \mathrm{m}, 4-\mathrm{H}\right.$ and $\left.\mathrm{OCH}_{2} \mathrm{CH}_{2} \mathrm{Si}\right), 4.46\left(2 \mathrm{H}, \mathrm{m}, \mathrm{PhCH}_{2} \mathrm{O}\right), 4.65\left(2 \mathrm{H}, \mathrm{s}, \mathrm{OCH}_{2} \mathrm{O}\right), 4.90(1 \mathrm{H}$, $\mathrm{m}, 1-\mathrm{H})$ and $7.30(5 \mathrm{H}, \mathrm{m}, \mathrm{Ph}-\mathrm{H})$; $\delta \mathrm{C}-1.5,13.4,14.1,18.0,18.4,21.0,26.5,29.8,36.3,36.8$, 65.0, 72.0, 72.9, 75.6, 76.6, 93.3, 127.3, 127.5, 128.2, 138.3 and 170.5; $\mathrm{m} / \mathrm{z}$ (C.I.) $470\left(\mathrm{M}^{+}+18\right.$, $100 \%), 263(34), 185(24)$ and $90(22)$.

\section{[2-(\{[(1R,4S,5S)-6-(Benzyloxy)-4-iodo-5-methyl-1-propylhexyl]oxy\}methoxy)-ethyl]}

(trimethyl)-silane (13). Imidazole (86 mg, $1.26 \mathrm{mmol}$ ), triphenylphosphine (200 $\mathrm{mg}$, $0.761 \mathrm{mmol})$ and iodine $(193 \mathrm{mg}, 0.761 \mathrm{mmol})$ were added to a solution of alcohol 9 (260 $\mathrm{mg}$, $0.634 \mathrm{mmol})$ in THF $\left(1 \mathrm{~cm}^{3}\right)$ at room temperature. The resultant suspension was stirred at room temperature for $15 \mathrm{~h}$. Saturated sodium hydrogen carbonate solution $\left(2 \mathrm{~cm}^{3}\right)$ was added and the mixture stirred for 10 mins. Iodine was then added until the organic layer remained iodine coloured. After a further 10 mins stirring saturated sodium thiosulfate solution $\left(5 \mathrm{~cm}^{3}\right)$ was added. The two layers were separated and the aqueous layer was extracted with ether $\left(3 \times 5 \mathrm{~cm}^{3}\right)$. The combined organic layers were washed with brine $\left(5 \mathrm{~cm}^{3}\right)$, dried $\left(\mathrm{MgSO}_{4}\right)$ and the solvent was removed under reduced pressure. The residue was preabsorbed onto silica and purified by flash chromatography using light petroleum : ether, $(4: 1)$ as eluant to afford the title compound, 13 (210 mg, 64\%), as a colourless oil, (Found: $\mathrm{M}^{+}+\mathrm{NH}_{4}, 538.2217 . \mathrm{C}_{23} \mathrm{H}_{45} \mathrm{NO}_{3} \mathrm{SiI}$ requires $M$, 538.2215); $v_{\max } / \mathrm{cm}^{-1} 1454,1375,1248,1099,1052,1031,858,835$ and $696 ; \delta_{\mathrm{H}} 0.00(9 \mathrm{H}, \mathrm{s}$, $\left.\left(\mathrm{CH}_{3}\right)_{3} \mathrm{Si}\right), 0.90\left(8 \mathrm{H}, \mathrm{m}, 5-\mathrm{CH}_{3}, \mathrm{CH}_{3} \mathrm{CH}_{2} \mathrm{CH}_{2}\right.$ and $\left.\mathrm{CH}_{2} \mathrm{Si}\right), 1.10-2.20\left(9 \mathrm{H}, \mathrm{m}, 2-\mathrm{H}_{2}, 3-\mathrm{H}_{2}, 5-\mathrm{H}\right.$ and $\left.\mathrm{CH}_{2} \mathrm{CH}_{2} \mathrm{CH}_{3}\right), 3.20-3.70\left(5 \mathrm{H}, \mathrm{m}, 4-\mathrm{H}, 6-\mathrm{H}_{2}\right.$ and $\left.\mathrm{OCH}_{2} \mathrm{CH}_{2} \mathrm{Si}\right), 4.49(3 \mathrm{H}, \mathrm{m}, 1-\mathrm{H}$ and $\left.\mathrm{PhCH}_{2} \mathrm{O}\right), 4.66\left(2 \mathrm{H}, \mathrm{s}, \mathrm{OCH}_{2} \mathrm{O}\right)$ and $7.26-7.36(5 \mathrm{H}, \mathrm{m}, \mathrm{Ph}-\mathrm{H}) ; \delta \mathrm{C}-1.4,14.1,14.2,18.0,18.5$, $34.3,36.4,39.4,46.6,65.1,73.2,75.6,76.0,93.4,127.5,127.6,128.2$ and $138.2 ; \mathrm{m} / \mathrm{z}$ (C.I.) 538 $\left(\mathrm{M}^{+}+18,100 \%\right), 410(58), 263(61)$ and $90(63)$.

(4R,7S,8S)-9-(Benzyloxy)-7-iodo-8-methylnonan-4-ol (14) and (2R,5R)-2-[(1S)-2-(Benzyloxy-1methylethyl]-5-propyltetrahydrofuran (15). Hydrogen fluoride $\left(0.30 \mathrm{~cm}^{3}, 40 \%\right.$ solution in water) was added to a solution of iodide $\mathbf{1 3}(154 \mathrm{mg}, 0.296 \mathrm{mmol})$ in acetonitrile $\left(1 \mathrm{~cm}^{3}\right)$. The resultant solution was stirred at room temperature for $15 \mathrm{~h}$. Saturated sodium hydrogen carbonate solution $\left(2 \mathrm{~cm}^{3}\right)$ was added. The aqueous phase was extracted with ether $\left(3 \times 3 \mathrm{~cm}^{3}\right)$ and the combined organic phase was washed with brine $\left(3 \mathrm{~cm}^{3}\right)$, dried $\left(\mathrm{MgSO}_{4}\right)$ and the solvent was removed under reduced pressure. Flash chromatography of the residue using light petroleum : ether, (4:1) gave title compound 14, (65 mg, 57\%), as a colourless oil and title compound 15, (14 $\mathrm{mg}, 18 \%)$, as a colourless oil. 
14. (Found: $\mathrm{M}^{+}+\mathrm{H}, 391.1139 . \mathrm{C}_{17} \mathrm{H}_{28} \mathrm{O}_{2} \mathrm{I}$ requires $M, 391.1135$ ); $v_{\max } / \mathrm{cm}^{-1} 3422,1453,1375$, $1364,1329,1204,1098,1026,738$ and $697 ; \delta_{\mathrm{H}} 0.96\left(6 \mathrm{H}, \mathrm{m}, 1-\mathrm{H}_{3}\right.$ and $\left.8 \mathrm{CH}_{3}\right), 1.20-2.20(9 \mathrm{H}$, m, 2- $\mathrm{H}_{2}, 3-\mathrm{H}_{2}, 5-\mathrm{H}_{2}, 6-\mathrm{H}_{2}$ and 8-H), $3.31-3.80\left(3 \mathrm{H}, \mathrm{m}, 7-\mathrm{H}\right.$ and 9- $\left.\mathrm{H}_{2}\right), 4.55(3 \mathrm{H}, \mathrm{m}, 4-\mathrm{H}$ and $\left.\mathrm{PhCH}_{2} \mathrm{O}\right)$ and $7.38(5 \mathrm{H}, \mathrm{m}, \mathrm{Ph}-\mathrm{H})$; $\delta \mathrm{C} \mathrm{13.9,} \mathrm{14.3,} \mathrm{18.7,} \mathrm{34.6,} \mathrm{37.4,} \mathrm{39.5,} \mathrm{39.7,} \mathrm{46.4,} \mathrm{70.6,} \mathrm{73.2,}$ 75.4, 127.5, 127.6, 128.3 and 138.2; $\mathrm{m} / \mathrm{z}$ (C.I.) $408\left(\mathrm{M}^{+}+18,40 \%\right), 391\left(\mathrm{M}^{+}+1,29\right), 263(100)$ and 106 (72).

15. (Found: $\mathrm{M}^{+}+\mathrm{H}, 263.2015 . \mathrm{C}_{17} \mathrm{H}_{27} \mathrm{O}_{2}$ requires $M, 263.2010$ ); $v_{\max } / \mathrm{cm}^{-1} 1455,1366,1100$, 1074, 735 and 697; $\delta \mathrm{H} 0.86\left(6 \mathrm{H}, \mathrm{m}, 1^{\prime}-\mathrm{CH}_{3}\right.$ and $\left.\mathrm{CH}_{3} \mathrm{CH}_{2} \mathrm{CH}_{2}\right), 1.20-1.90\left(9 \mathrm{H}, \mathrm{m}, 1\right.$ ' $-\mathrm{H}, 3-\mathrm{H}_{2}$, 4- $\mathrm{H}_{2}$ and $\left.\mathrm{CH}_{2} \mathrm{CH}_{2} \mathrm{CH}_{3}\right), 3.29$ (1 H, m, 2'-H), 3.54 (1 H, dd, $J 4$ and 9, 2'- $\mathrm{H}$ ), 3.62 (1 H, m, 2-H), $3.73(1 \mathrm{H}, \mathrm{m}, 5-\mathrm{H}), 4.42\left(2 \mathrm{H}, \mathrm{s}, \mathrm{PhCH}_{2} \mathrm{O}\right)$ and $7.26(5 \mathrm{H}, \mathrm{m}, \mathrm{Ph}-\mathrm{H}) ; \delta \mathrm{C} 13.4,14.1,19.3,28.4$, $30.8,38.2,38.8,72.9,73.2,78.9,80.6,127.2,127.3,128.1$ and 138.8; m/z (C.I.) $280(2 \%), 263$ (100) and $173(5)$.

Alcohol (14). (62 mg, $0.159 \mathrm{mmol})$ in THF $\left(0.5 \mathrm{~cm}^{3}\right)$ was added to a suspension of sodium hydride $(8 \mathrm{mg}, 60 \%$ dispersion in mineral oil, $0.206 \mathrm{mmol})$ in THF $\left(0.5 \mathrm{~cm}^{3}\right)$ at $0{ }^{\circ} \mathrm{C}$. The resultant suspension was stirred at room temperature for $3 \mathrm{~h}$. Water $\left(1 \mathrm{~cm}^{3}\right)$ was added and the aqueous phase was extracted with ether $\left(3 \times 3 \mathrm{~cm}^{3}\right)$. The combined organic phase was washed with brine $\left(4 \mathrm{~cm}^{3}\right)$, dried $\left(\mathrm{MgSO}_{4}\right)$ and the solvent was removed under reduced pressure. Flash chromatography of the residue using light petroleum : ethyl acetate, $(20: 1)$ yielded the title compound, 15 (27 mg, 65\%), as a colourless oil, (Found: $\mathrm{M}^{+}+\mathrm{H}, 263.2015 . \mathrm{C}_{17} \mathrm{H}_{27} \mathrm{O}_{2}$ requires $M$, 263.2010); $v_{\max } / \mathrm{cm}^{-1} 1455,1365,1100,1074,735$ and 697. The ${ }^{1} \mathrm{H}$ and ${ }^{13} \mathrm{C}$ spectroscopic data were identical to that for the tetrahydrofuran obtained directly from the deprotection.

( \pm )-1-Benzyloxy-3-mesyloxy-2-methyl-6-(2-trimethylsilylethoxy)methoxynonane (16). To a stirred solution of the alcohol $9(201 \mathrm{mg}, 0.49 \mathrm{mmol})$ and triethylamine $(204 \mu \mathrm{L}, 1.47 \mathrm{mmol})$ in $\operatorname{DCM}\left(5 \mathrm{~cm}^{3}\right)$ at $0{ }^{0} \mathrm{C}$, methanesulphonyl chloride $(114 \mu \mathrm{L}, 1.47 \mathrm{mmol})$ was added dropwise. The mixture was stirred at $0{ }^{0} \mathrm{C}$ for $1 \mathrm{~h}$, then saturated aqueous $\mathrm{NaHCO}_{3}\left(5 \mathrm{~cm}^{3}\right)$ was added and the mixture allowed to warm to ambient temperature. The reaction mixture was partitioned between dichloromethane $\left(10 \mathrm{~cm}^{3}\right)$ and water $\left(10 \mathrm{~cm}^{3}\right)$. The aqueous layer was extracted with dichloromethane $\left(2 \times 10 \mathrm{~cm}^{3}\right)$ and the combined organic phase was washed with brine $(2 \mathrm{x}$ $\left.15 \mathrm{~cm}^{3}\right)$, dried $\left(\mathrm{MgSO}_{4}\right)$ and the solvent removed under reduced pressure to give an oil. Flash chromatography using petrol : ether (20:1) as eluent gave the title compound, 16 (145 mg, 61\%), (Found: 506.2979. $\mathrm{C}_{24} \mathrm{H}_{44} \mathrm{O}_{6} \mathrm{SSi}$ requires 506.2971); $v_{\max } / \mathrm{cm}^{-1} 2934,1455,1354,1333,1175$, 1099, 1029, 907, 736 and 699; $\delta_{\mathrm{H}} 0.00\left(9 \mathrm{H}, \mathrm{s}, \mathrm{SiMe}_{3}\right), 0.90\left(5 \mathrm{H}, \mathrm{m}, 9-\mathrm{CH}_{3}, \mathrm{SiCH}_{2}\right), 0.99(3 \mathrm{H}$, $\left.\mathrm{d}, J=7.01 \mathrm{~Hz}, 2-\mathrm{CH}_{3}\right), 1.26-1.52\left(4 \mathrm{H}, \mathrm{m}, 7-\mathrm{CH}_{2}, 8-\mathrm{CH}_{2}\right), 1.56-1.75\left(4 \mathrm{H}, \mathrm{m}, 4-\mathrm{CH}_{2}, 5-\mathrm{CH}_{2}\right)$, 2.28 (1 H, m, 2-CH), 2.97 (3 H, s, SO2CH3), 3.40 (2 H, m, 1-CH2), 3.58 (3 H, m, 6-H, $\mathrm{OCH}_{2} \mathrm{CH}_{2} \mathrm{SiMe}_{3}$ ), 4.44 and 4.52 (each $1 \mathrm{H}, \mathrm{d}, \mathrm{J}=11.95 \mathrm{~Hz}, \mathrm{OCH}_{2} \mathrm{Ar}$ ), $4.66\left(2 \mathrm{H}, \mathrm{s}, \mathrm{O}-\mathrm{CH}_{2}-\mathrm{O}\right)$, $4.81(1 \mathrm{H}, \mathrm{m}, 3-\mathrm{H})$ and $7.22-7.44(5 \mathrm{H}, \mathrm{m}, \mathrm{ArH})$; $\delta \mathrm{C}-1.5,12.6,14.1,18.0,18.5,26.1,29.3$, $36.3,37.2,38.4,65.1,71.4,73.0,73.3,85.3,93.4,127.5,127.7,128.3$ and $137.2 ; \mathrm{m} / \mathrm{z}$ (C.I.) 506 (20\%), 404 (20) and 263 (100). 
2,5-trans-3-Hydroxy-5-propyl-2-[(1'-methyl-2-tert-butyldimethylsilyloxy)ethyl]tetrahydrofuran (18) and 2,5-trans-3-Hydroxy-5-propyl-2-[(1'-methyl-2-benzyloxy)ethyl]tetrahydrofuran (19). To a stirred solution of epoxide $7(189 \mathrm{mg}, 0.679 \mathrm{mmol})$ in dichloromethane $\left(5 \mathrm{~cm}^{3}\right)$, tert-butyl dimethylsilyl trifluoromethanesulphonate $(155 \mu \mathrm{L}, 0.679 \mathrm{mmol})$ and trimethylaluminium $(679 \mu \mathrm{L}, 1.0 \mathrm{M}$ in hexane, $0.679 \mathrm{mmol})$ were added at $0{ }^{0} \mathrm{C}$. The reaction mixture was stirred at room temperature for $16 \mathrm{~h}$, then a mixture of acetic acid and water ( 3 $\mathrm{cm}^{3}: 1.5 \mathrm{~cm}^{3}$ ) was added at $0{ }^{0} \mathrm{C}$. The mixture was allowed to stir at room temperature for a further $16 \mathrm{~h}$. Water $\left(15 \mathrm{~cm}^{3}\right)$ was added and the mixture partitioned between dichloromethane $\left(10 \mathrm{~cm}^{3}\right)$ and water. The aqueous layer was extracted with dichloromethane $\left(2 \times 5 \mathrm{~cm}^{3}\right)$ and the combined organic phase washed with water $\left(2 \times 20 \mathrm{~cm}^{3}\right)$, brine $\left(2 \times 20 \mathrm{~cm}^{3}\right)$ and dried $\left(\mathrm{MgSO}_{4}\right)$. After concentration under reduced pressure, flash chromatography of the residue using petrol : ether (10:1) as eluent afforded the title compound $\mathbf{1 8}(45 \mathrm{mg}, 22 \%)$. Further elution using petrol : ether (5:2) as eluent afforded the title compound 19 (119 $\mathrm{mg}, 63 \%)$.

18. (Found: $(\mathrm{M}+1)^{+}, 303.2355 . \mathrm{C}_{16} \mathrm{H}_{35} \mathrm{O}_{3} \mathrm{Si}$ requires $M$, 303.2355); $v_{\max } / \mathrm{cm}^{-1} 3473,2930,1463$, 1254, 1099, 836, 776 and 696; $\delta_{\mathrm{H}} 0.00\left(6 \mathrm{H}, \mathrm{s}, \mathrm{SiMe}_{2}\right), 0.85\left(12 \mathrm{H}, \mathrm{m}, \mathrm{CH}_{3}, \mathrm{CMe}_{3}\right), 0.92(3 \mathrm{H}, \mathrm{d}$, $\left.J=6.87 \mathrm{~Hz}, 1^{\prime}-\mathrm{CH}_{3}\right), 1.35\left(5 \mathrm{H}, \mathrm{m}, 4-\mathrm{H}, 2 \mathrm{x} \mathrm{CH}_{2}\right), 1.62\left(1 \mathrm{H}, \mathrm{dt}, J=2.33 \mathrm{~Hz} \& 14.01 \mathrm{~Hz}, 4-\mathrm{H}^{\prime}\right)$, $1.77(1 \mathrm{H}, \mathrm{br} \mathrm{s}, \mathrm{OH}), 2.16(1 \mathrm{H}, \mathrm{m}, 1-\mathrm{CH}), 3.46(1 \mathrm{H}, \mathrm{t}, J=8.51 \mathrm{~Hz}, 5-\mathrm{H}), 3.68\left(1 \mathrm{H}, \mathrm{s}, 2^{\prime}-\mathrm{H}\right)$, $3.75(1 \mathrm{H}, \mathrm{dd}, J=3.29 \mathrm{~Hz} \& 5.63 \mathrm{~Hz}, 2-\mathrm{H}), 3.84(1 \mathrm{H}, \mathrm{dt}, J=2.89 \mathrm{~Hz} \& 11.12 \mathrm{~Hz}, 3-\mathrm{H})$ and $3.93\left(1 \mathrm{H}, \mathrm{t}, J=9.89 \mathrm{~Hz}, 2^{\prime}-\mathrm{H}\right)$; $\delta \mathrm{C} 4.7,10.6,14.0,18.0,18.5,25.7,37.4,39.6,40.2,71.3,73.4$, 78.8 and $86.8 ; \mathrm{m} / \mathrm{z}$ (C.I.) $320(2 \%)$ and $303(100)$.

19. (Found: $(\mathrm{M}+1)^{+}, 279.1954 . \mathrm{C}_{17} \mathrm{H}_{27} \mathrm{O}_{3}$ requires $\left.M, 279.1960\right) ; v_{\max } / \mathrm{cm}^{-1} 3468,2931,1454$, 1357, 1308, 1095, 736 and 698; $\delta_{\mathrm{H}} 0.86\left(3 \mathrm{H}, \mathrm{t}, J=6.18 \mathrm{~Hz}, \mathrm{CH}_{3}\right), 1.02\left(3 \mathrm{H}, \mathrm{d}, J=6.87 \mathrm{~Hz}, 1^{\prime}-\right.$ $\left.\mathrm{CH}_{3}\right), 1.37\left(5 \mathrm{H}, \mathrm{m}, 4-\mathrm{H}, 2 \times \mathrm{CH}_{2}\right), 1.58\left(1 \mathrm{H}, \mathrm{dt}, J=2.85 \mathrm{~Hz} \& 14.15 \mathrm{~Hz}, 4-\mathrm{H}^{\prime}\right), 2.29(1 \mathrm{H}, \mathrm{m}, 1-$ $\mathrm{CH}), 3.49(1 \mathrm{H}, \mathrm{m}, 5-\mathrm{H}), 3.55\left(1 \mathrm{H}, \mathrm{dd}, J=3.98 \mathrm{~Hz} \& 6.32 \mathrm{~Hz}, 2^{\prime}-\mathrm{H}\right), 3.74(1 \mathrm{H}, \mathrm{m}, 3-\mathrm{H}), 3.95(1$ $\mathrm{H}, \mathrm{dd}, J=7.96 \mathrm{~Hz} \& 15.24 \mathrm{~Hz}, 2-\mathrm{H}), 4.03\left(1 \mathrm{H}, \mathrm{dt}, J=3.70 \mathrm{~Hz} \& 9.57 \mathrm{~Hz}, 2-\mathrm{H}^{\prime}\right), 4.48(2 \mathrm{H}, \mathrm{s}$, $\left.\mathrm{OCH}_{2} \mathrm{Ar}\right)$ and $7.00-7.38(5 \mathrm{H}, \mathrm{m}, \mathrm{ArH}) ; \delta \mathrm{C} 10.7,14.0,18.5,36.2,39.6,40.8,71.1,71.8,73.6$, 83.2, 85.0, 127.5, 127.7, 128.4 and 138.0; $\mathrm{m} / \mathrm{z}$ (C.I.) $296(8 \%)$ and $279(100)$.

\section{2,5-trans-3-Hydroxy-5-propyl-2-[(1'-methyl-2-hydroxy)ethyl]tetrahydrofuran (20)}

Method A : By debenzylation of 19 : Palladium on charcoal $(10 \%, 10 \mathrm{mg})$ and 5 drops of acetic acid were added to a solution of tetrahydrofuran, 19 (46 mg, $0.165 \mathrm{mmol})$ in absolute ethanol $\left(3 \mathrm{~cm}^{3}\right)$. The flask was evacuated and flushed with hydrogen four times, and then the reaction mixture was allowed to stir under an atmosphere of hydrogen for $72 \mathrm{~h}$. The mixture was filtered through Celite and the residue washed with ethanol $\left(2 \times 5 \mathrm{~cm}^{3}\right)$. The filtrate was extracted with dichloromethane $\left(2 \times 5 \mathrm{~cm}^{3}\right)$ and the organic phase washed with water $\left(2 \times 10 \mathrm{~cm}^{3}\right)$, brine $(2 \times 10$ $\left.\mathrm{cm}^{3}\right)$ then dried $\left(\mathrm{MgSO}_{4}\right)$. Concentration under reduced pressure gave an oil which on flash chromatography using petrol : ether (1:1) as eluent gave the title compound, 20 (85 mg, 91\%), (Found: $(\mathrm{M}+1)^{+}, 189.1489 . \mathrm{C}_{10} \mathrm{H}_{21} \mathrm{O}_{3}$ requires $\left.M, 189.1490\right) ; v_{\max } / \mathrm{cm}^{-1} 3399,3366,2960,1459$, 1127, 1054, 996 and 815; $\delta_{\mathrm{H}} 0.86\left(3 \mathrm{H}, \mathrm{t}, J=6.73 \mathrm{~Hz}, \mathrm{CH}_{3}\right), 0.97\left(3 \mathrm{H}, \mathrm{d}, J=7.01 \mathrm{~Hz}, 1^{\prime}-\mathrm{CH}_{3}\right)$, $1.36\left(5 \mathrm{H}, \mathrm{m}, 4-\mathrm{H}, 2 \times \mathrm{CH}_{2}\right), 1.68(1 \mathrm{H}, \mathrm{m}, 4-\mathrm{H}), 1.92(1 \mathrm{H}, \mathrm{d}, J=4.39 \mathrm{~Hz}, 3 \mathrm{OH}), 2.24(1 \mathrm{H}, \mathrm{m}$, 1'-CH), $3.44(2 \mathrm{H}, \mathrm{m}, 5-\mathrm{H}, 2-\mathrm{OH}), 3.81(2 \mathrm{H}, \mathrm{m}, 2-\mathrm{H}, 2-\mathrm{H}), 3.91(1 \mathrm{H}, \mathrm{dt}, J=3.71 \mathrm{~Hz} \& 10.03$ 
$\mathrm{Hz}, 3-\mathrm{H})$ and $3.97(1 \mathrm{H}, \mathrm{t}, J=7.97 \mathrm{~Hz}, 2$-H); $\delta \mathrm{C} 9.9,14.0,18.6,37.2,40.2,71.1,73.1,78.1$ and 86.3; $\mathrm{m} / \mathrm{z}$ (C.I.) 206 (15\%) and 189 (100).

Method B : By deprotection of 18 : TBAF ( $645 \mu \mathrm{L}, 0.645 \mathrm{mmol})$ was added to a solution of the silyl ether, $18(65 \mathrm{mg}, 0.215 \mathrm{mmol})$ in THF $\left(2 \mathrm{~cm}^{3}\right)$ at $0{ }^{0} \mathrm{C}$. After $5 \mathrm{~h}$ at room temperature, water $\left(5 \mathrm{~cm}^{3}\right)$ was added. The reaction mixture was extracted with ether $\left(2 \times 10 \mathrm{~cm}^{3}\right)$ the organic phase washed with brine $\left(2 \times 10 \mathrm{~cm}^{3}\right)$ then dried $\left(\mathrm{MgSO}_{4}\right)$.

Concentration under reduced pressure gave an oil which on flash chromatography using petrol : ether (1:1) as eluent gave the diol 20 (36 mg, 89\%).

\section{2,5-trans-3-O-Phenoxythiocarbonyl-5-propyl-2-[(1'-methyl-2-benzyloxy)ethyl]-}

tetrahydrofuran (21). To a stirred solution of tetrahydrofuran 19 (37 $\mathrm{mg}, 0.133 \mathrm{mmol})$ in dichloromethane $\left(2 \mathrm{~cm}^{3}\right)$ was added pyridine $(43 \mu \mathrm{L}, 0.532 \mathrm{mmol})$ and phenoxythiocarbonyl chloride $(20 \mu \mathrm{L}, 0.146 \mathrm{mmol})$. After $3 \mathrm{~h}$, water $\left(3 \mathrm{~cm}^{3}\right)$ was added and the mixture partitioned between water $\left(5 \mathrm{~cm}^{3}\right)$ and dichloromethane $\left(5 \mathrm{~cm}^{3}\right)$. The organic phase was washed with brine $\left(2 \times 5 \mathrm{~cm}^{3}\right)$ dried $\left(\mathrm{MgSO}_{4}\right)$ and concentrated under reduced pressure to afford an oil which on flash chromatography using petrol : ether (10:1) gave the title compound, 21 (46 mg, 83\%); (Found: $(\mathrm{M}+18)^{+}, 432.1997 . \mathrm{C}_{24} \mathrm{H}_{34} \mathrm{NO}_{4} \mathrm{~S}$ requires $\left.M, 432.1993\right) ; v_{\max } / \mathrm{cm}^{-1} 2960,1593,1494$, $1455,1256,1210,1093,777,734$ and 698; $\delta_{\mathrm{H}} 0.90\left(3 \mathrm{H}, \mathrm{t}, J=7.14 \mathrm{~Hz}, \mathrm{CH}_{3}\right), 1.04(3 \mathrm{H}, \mathrm{d}, J=$ $\left.6.87 \mathrm{~Hz}, 1^{\prime}-\mathrm{CH}_{3}\right), 1.38\left(2 \mathrm{H}, \mathrm{t}, J=7.14 \mathrm{~Hz}, \mathrm{CH}_{2}\right), 1.52(1 \mathrm{H}, \mathrm{m}), 1.62(1 \mathrm{H}, \mathrm{m}), 1.75(1 \mathrm{H}, \mathrm{dd}, J=$ $5.35 \mathrm{~Hz} \& 10.43 \mathrm{~Hz}, 4-\mathrm{H}), 1.78\left(1 \mathrm{H}, \mathrm{m}, 4-\mathrm{H}^{\prime}\right), 2.28(1 \mathrm{H}, \mathrm{m}, 1-\mathrm{CH}), 3.48(1 \mathrm{H}, \mathrm{t}, J=8.37 \mathrm{~Hz}, 5-$ H), 3.59 (1 H, dd, $J=7.05 \mathrm{~Hz} \& 10.03 \mathrm{~Hz}, 2-\mathrm{H}), 3.98$ (2 H, m, 2'-H, 2-H), 4.50 (2 H, s, $\left.\mathrm{OCH}_{2} \mathrm{Ar}\right), 4.92(1 \mathrm{H}, \mathrm{m}, 3-\mathrm{H})$ and 7.04-7.38 (10 H, m, ArH); $\delta \mathrm{C} 1.0,10.7,14.1,18.6,36.3,39.6$, $40.8,71.1,71.8,73.6,83.2,85.0,121.8,126.7,127.6,127.7,128.4,129.4,129.6$ and $153.5 ; \mathrm{m} / \mathrm{z}$ (C.I.) 432 (80\%), 414 (50), 398 (100) and 261 (20).

2,5-trans-5-Propyl-2-[(1'-methyl-2-benzyloxy)ethyl]tetrahydrofuran (17). To a stirred solution of tetrahydrofuran $21(42 \mathrm{mg}, 0.101 \mathrm{mmol})$ in toluene $\left(1.5 \mathrm{~cm}^{3}\right)$ was added tri-n-butyltin hydride $(27 \mu \mathrm{L}, 0.101 \mathrm{mmol})$ and AIBN $(5 \mathrm{mg})$. The solution was heated under reflux under a nitrogen atmosphere for $2 \mathrm{~h}$ then concentrated under reduced pressure. Flash chromatography of the residue using petrol : ether (20:1) as eluent gave the title compound, 17 (14 mg, 53\%), as a colourless oil, (Found: $(\mathrm{M}+18)^{+}, 280.2270 . \mathrm{C}_{17} \mathrm{H}_{30} \mathrm{NO}_{2}$ requires $\left.M, 280.2276\right) ; v_{\max } / \mathrm{cm}^{-1} 2929$, $1495,1455,1257,1095,784$ and 697; $\delta_{\mathrm{H}} 0.81\left(1 \mathrm{H}, \mathrm{t}, J=6.45 \mathrm{~Hz}, \mathrm{CH}_{3}\right), 1.01(1 \mathrm{H}, \mathrm{d}, J=6.86$ $\left.\mathrm{Hz}, 1^{\prime}-\mathrm{CH}_{3}\right), 1.22\left(6 \mathrm{H}, \mathrm{m}, 3-\mathrm{H}, 4-\mathrm{H}, 2 \mathrm{x} \mathrm{CH}_{2}\right), 1.36\left(2 \mathrm{H}, \mathrm{m}, 3-\mathrm{H}^{\prime}, 4-\mathrm{H}\right), 2.23$ (1 H, m, 1-CH), $3.44(1 \mathrm{H}, \mathrm{q}, J=8.38 \mathrm{~Hz}, 2-\mathrm{H}), 3.51(1 \mathrm{H}, \mathrm{dd}, J=6.04 \mathrm{~Hz} \& 9.47 \mathrm{~Hz}, 2-\mathrm{H}), 3.82$ (1 H, q, $J=$ $6.86 \mathrm{~Hz}, 5-\mathrm{H}), 3.90(1 \mathrm{H}, \mathrm{dd}, J=7.14 \mathrm{~Hz} \& 8.10 \mathrm{~Hz}, 2 /-\mathrm{H}$ ), 4.43 and 4.47 (each $1 \mathrm{H}, \mathrm{d}, J=11.94$ $\left.\mathrm{Hz}, \mathrm{OCH}_{2} \mathrm{Ar}\right)$ and 7.15-7.35 (5 H, m, ArH); $\delta \mathrm{C} \mathrm{10.4,} \mathrm{14.0,} \mathrm{22.5,} \mathrm{25.7,} \mathrm{31.8,} \mathrm{34.6,} \mathrm{37.0,} \mathrm{71.5,}$ 73.2, 83.4, 84.5, 127.5, 127.6, 128.3 and 138.4; $\mathrm{m} / \mathrm{z}$ (C.I.) $280(100 \%)$ and 263 (85).

Aqueous hydrogen fluoride $\left(40 \%, 8 \mathrm{~cm}^{3}\right)$ and acetonitrile (5 drops) were added to the SEMether 16 (97 mg, $0.2 \mathrm{mmol})$, and the reaction mixture was stirred at room temperature for $6 \mathrm{~h}$. The reaction was quenched by the addition of water $\left(5 \mathrm{~cm}^{3}\right)$ and extracted with ether $(2 \mathrm{x}$ $\left.10 \mathrm{~cm}^{3}\right)$. The organic phase was washed with brine $\left(2 \times 10 \mathrm{~cm}^{3}\right)$, dried $\left(\mathrm{MgSO}_{4}\right)$ and 
concentrated under reduced pressure to give an oil. Flash chromatography using petrol : ether (20:1) as eluent gave the tetrahydrofuran $\mathbf{1 7}$ identical to a sample prepared from the thionocarbonate 21 (16.1 $\mathrm{mg}, 31 \%)$.

\section{Acknowledgements}

We thank the University of Manchester and GlaxoSmithKline for a studentship (to L. A. H.) under the CASE scheme and the Commonwealth Scholarship Commission for a Fellowship (to L. W. S.).

\section{References and Notes}

1. (a) Kondo, S.; Yasui, K.; Natsume, M.; Katayama, M.; Marumo, S. J. Antibiot. 1988, 41, 1196. (b) Kondo, S.; Yasui, K.; Katayama, M.; Marumo, S.; Kondo, T.; Hattori, H. Tetrahedron Lett. 1987, 28, 5861. (c) Natsume, M.; Kondo, S.; Marumo, S. J. Chem. Soc., Chem. Commun. 1989, 1911.

2. Solladie, G.; Dominguez, C. J. Org. Chem. 1994, 59, 3898.

3. Thomas, E.; J. J. Chem. Soc., Chem. Commun. 1997, 411.

4. Arista, L.; Gruttadauria, M.; Thomas, E. J. Synlett 1997, 627.

5. Mihelich, E. D. J. Amer. Chem. Soc. 1990, 112, 8995.

6. Kumar, N.; Thomas, E. J. unpublished information.

7. (a) Kang, S. H.; Hwang, T. S.; Kim, W. J.; Lim, J. K. Tetrahedron Lett. 1990, 31, 5917. (b) Kang, S. H.; Hwang, T. S.; Kim, W. J.; Lim, J. K. Tetrahedron Lett. 1991, 32, 4015. (c) Ley, S. V.; Lygo, B. Tetrahedron Lett. 1982, 23, 4625. (d) Brussani, G.; Ley, S. V.; Wright, J. L.; Williams, D. J. J. Chem. Soc., Perkin Trans. I 1986, 303.

8. Mihelich, D.; Hite, G. A. J. Amer. Chem. Soc. 1992, 114, 7318.

9. Kumar, N.; Thomas, E. J. submitted to Tetrahedron Lett.

10. 10) Mihelich, E. D.; Daniels, K.; Eickhoff, D. J. J. Am. Chem. Soc. 1982, 103, 7690.

11. Jung, M. E.; D’Amico, D. C. J. Am. Chem. Soc. 1997, 119, 12150. 